\title{
A Randomized Controlled Trial of a Tailored Primary Care Program to Reverse Excessive Alcohol Consumption
}

\author{
Mirrian Hilbink, PhD, Gerlienke Voerman, PhD, Ivonne van Beurden, MSc, \\ Brenda Penninx, PhD and Miranda Laurant, PhD
}

Objectives: To assess the effects of a tailored, multifaceted intervention in primary care on the level of patients' alcohol consumption and to investigate which patient and organizational factors determine a reduction in alcohol consumption.

Methods: This was a cluster randomized, controlled trial conducted among primary care practices in The Netherlands. Data from 6318 patients were available, of whom 712 patients from 70 practices were hazardous or harmful alcohol users. The improvement (intervention) program combined professional, organizational, and patient-directed activities. The emphasis was on educational training for general practitioners and support visits by a trained facilitator, tailored to the participants' needs and attitudes. The primary outcome was the proportion of patients with hazardous or harmful alcohol consumption, as measured with the Alcohol Use Disorders Identification Test, who reduced their levels of alcohol consumption to low-risk levels after 2 years.

Results: Of the hazardous and harmful alcohol users, a substantial proportion (41.6\%) reduced their alcohol consumption to a low-risk level. The trial revealed a significant difference in favor of the control group: $35.5 \%$ of the patients with hazardous and harmful alcohol consumption in general practitioners' practices in the intervention group and $47.0 \%$ of this patient group in general practitioners' practices in the control group reduced their alcohol consumption to a low-risk level (odds ratio [OR], 0.62; 95\% confidence interval [CI], 0.43-0.90). Older age (OR, $1.02 ; 95 \% \mathrm{CI}, 1.01-1.03)$, female sex (OR, 2.00; 95\% CI, 1.26-3.19), and attitudes toward alcohol use seemed to be the most important predictors for the reduction of alcohol use to a low-risk level. Patients who considered it important to reduce alcohol consumption and patients who believed that less alcohol complicates relaxation were less likely to reduce their alcohol use to a low-risk level (OR, 0.39; 95\% CI, 0.19-0.80 and OR, 0.58; 95\% CI, 0.370.90, respectively). Characteristics of the general practices, however, were not associated with reduced alcohol use.

Conclusions: Our results suggest that the intervention has been counterproductive because the proportion of patients reducing their levels of alcohol consumption to low-risk levels was lower in the intervention group compared with the control group. Furthermore, our study demonstrated that patients' attitudes toward alcohol use are an important determinant of the success of the program. Therefore, future research should focus on the effectiveness of methods to change patients' attitudes. (J Am Board Fam Med 2012;25:712-722.)

Keywords: Alcohol Drinking, Alcoholism, Primary Health Care, Quality of Health Care, Randomized Controlled Trial

Excessive alcohol use is a major public health problem. In approximately 55 million adult Europeans

This article was externally peer reviewed.

Submitted 6 March 2012; revised 12 June 2012; accepted 19 June 2012.

From the Scientific Institute for Quality of Healthcare, Radboud University Nijmegen Medical Centre, Nijmegen, The Netherlands (AHWH, GEV, IB, BWJHP, MGHL); and the Department of Psychiatry, EMGO Institute, VU University Medical Center, Amsterdam, The Netherlands (BWJHP).

Funding: This study was supported by a grant from the Netherlands Organization for Health Research and Development (ZonMw), dossier no. 63000003.

Conflict of interest: none declared.
(15\% of the adult population), alcohol consumption is at least hazardous. ${ }^{1}$ It is a major risk factor

Ethical approval: The trial was approved by the research ethics committee CMO of the region Arnhem-Nijmegen (letter of 2 January 2006; SE/CMO 0003). The committee concludes in their letter that in compliance with the law on medical-scientific research (WMO), the GPA trial does not need approval. We asked written informed consent which indeed has been provided by all participants.

Corresponding author: Mirrian Hilbink, $\mathrm{PhD}$, Radboud University Nijmegen Medical Centre, IQ healthcare, PO Box 9101, Code: 114 IQ healthcare, 6500 HB Nijmegen, The Netherlands (E-mail: m.hilbink@iq.umcn.nl). 
for ill health and premature death and causes a large number of different types of diseases and injuries. Furthermore, alcohol is responsible for widespread social, mental, and emotional harms. ${ }^{2}$ Excessive alcohol use harms not only the user, but also those surrounding the user, including fetuses, family members, and those who suffer from crime, family violence, and driving accidents. ${ }^{3-5}$

Intervening in risky drinking patterns through screening and brief interventions (SBIs) at an early stage is a highly effective and cost-effective strategy to prevent drinking problems. ${ }^{6-8}$ Primary health care has been identified as a suitable setting for such interventions because of the high proportion of the total population seeking primary health care annually ${ }^{9}$ and because health promotion is a common and essential element of primary care. ${ }^{10}$

Research has shown that SBIs in primary health care settings are effective in reducing alcohol-related problems among persons with hazardous and harmful alcohol consumption but without alcohol dependence. $^{2}$ The latter patient category needs to be identified by the primary health care provider and subsequently referred to specialized mental health and addiction care for more intensive and specialized treatment. ${ }^{11}$ Despite its evidence, SBIs have not yet been integrated into the routine clinical practice of general practitioners (GPs). Primary health care workers often find it difficult to identify and advise patients in relation to alcohol use, most because of a lack of time, inadequate training, concern about antagonizing patients, and doubts about the effectiveness of SBIs. ${ }^{12-14}$

A systematic review of strategies to engage GPs in health promotion interventions showed that educational and office-based strategies increased GP involvement in SBIs by $13 \%$. Alcohol-specific and multifaceted strategies were the most promising. ${ }^{15}$ This is consistent with other implementation studies. ${ }^{16-18}$ However, to date very few studies of acceptable methodological quality with a reasonable follow-up period have tested the effectiveness of implementation strategies to engage GPs in the prevention of hazardous and harmful alcohol consumption. ${ }^{15}$ Furthermore, most implementation studies did not include outcomes of changes in patients' behavior. ${ }^{15}$ Therefore, we developed a multifaceted intervention on GPs' behavior toward prevention of hazardous and harmful alcohol consumption, and we conducted a randomized, controlled study to assess its 1-year effects on GPs' screening and advice-giving behavior and its 2-year effects on the level of alcohol consumption of patients. ${ }^{19}$ As reported in a previous article, ${ }^{19}$ our program did not result in improvement in rates of screening and giving of advice. In this article, we focus on the effect of our program on the level of alcohol consumption of patients. When considering the results of the previous article, ${ }^{19}$ a significant effect on patient outcome cannot reasonably be expected from our program. We therefore hypothesized that between GPs involved in the intervention program compared with those in the control group there will be no difference in the proportion of patients with hazardous and harmful alcohol consumption who reduced their levels of alcohol consumption to low-risk levels. In addition to the effect of our program on the level of alcohol consumption, we investigated which factors are associated with the reduction of alcohol consumption to a low-risk level. The current literature shows that the success of improvement programs can be determined by patient, professional, and organizational characteristics. ${ }^{20}$ To our knowledge, the variables associated with a reduction of alcohol consumption are yet unknown. By understanding these factors, strategies can be identified to adapt and focus future interventions.

\section{Methods}

\section{Design and Participants}

We performed a cluster randomized, controlled trial with measurements before (T0) and after (T1) the intervention. Patients were blinded to the GPs' participation in the study. Because GPs were aware of the intervention in which they took part, blinding of GPs was impossible. In total, 2758 Dutch general practices were invited to participate. Every GP within a practice individually received an invitation, but practices could only enroll if all GPs in the practice agreed to participate because the intervention contained several practice-directed elements. The enrolled practices were randomized by a computerized scheme such that the intervention and the control group would be of equal size. Randomization was performed before baseline measurement. To avoid possible bias, the GPs were informed about the group (intervention/control) to which they were assigned after obtaining baseline information. The intervention, described in the next section, was offered to the general practices during the period of October 2006 to June 2007 (intervention period). Details of both the random- 
ization procedure and the provision of the intervention have been described in detail elsewhere. ${ }^{19}$

\section{Intervention}

The improvement (intervention) program combined professional, organizational, and patient-directed activities. The emphasis was on educational training and support visits by a trained facilitator and were tailored to the participants' needs and attitudes. Patient awareness was, among other things, raised through personal feedback. A GP was as an advisor involved in the development of this program. For a detailed outline of the program, see the box. ${ }^{21,22}$

The control group was mailed the guidelines and patient information letters about problematic alcohol consumption (which can be considered usual care) but received no further support or training. For ethical reasons, their patients also received personal feedback on alcohol consumption in June 2007 after closure of the intervention period.

\section{Measures \\ Dependent Variable}

The primary outcome measure was the proportion of patients with hazardous and harmful alcohol consumption who reduced their levels of alcohol consumption to low-risk levels. Alcohol consumption was measured using the Alcohol Use Disorders Identification Test (AUDIT), a validated screening questionnaire developed to detect harmful and hazardous alcohol use. ${ }^{23}$ Each practice team was asked to hand out a written self-report AUDIT questionnaire to up to 230 consecutive patients who visited the general practice within a 3 -month period. The questionnaires were completed and returned to the research team before the start of the intervention. According to the published literature, ${ }^{23}$ the following cutoff AUDIT scores were used:

1. Score 0 to 7: Safe to moderate drinker

2. Score 8 to 15: Hazardous drinker

3. Score 16 to 19 : Harmful drinker

4. Score $\geq 20$ : Possibly dependent drinker

Patients with hazardous and harmful alcohol consumption $(7<$ AUDIT score $<20)$ were followed up by the research team 2 years after their first completion of the AUDIT questionnaire and were requested to complete the AUDIT questionnaire once again. Because our intervention aimed to reduce alcohol-related problems among persons with hazardous and harmful alcohol consumption without alcohol dependence, neither safe to moderate drinkers nor possibly dependent drinkers were followed up.

\section{Independent Variables}

Patient Characteristics. We collected patient characteristics from the individual self-administered questionnaires, simultaneously offered to patients in combination with the AUDIT questionnaire. Patient characteristics included age, sex, household composition (living alone/living with partner, children, or both); educational level (basic, intermediate, high); smoking status (yes/no); comorbidity; and attitudes toward alcohol use. Comorbidity was defined in terms of presence or absence of certain comorbid conditions, including hypertension, cardiovascular disease, stomach problems, liver disease, colon disease, cancer, stroke, diabetes, respiratory disease, depressive disorder, anxiety disorder, relation problems, substance or drug abuse, insomnia, and other chronic disease or problem. Patients' attitudes toward alcohol use were measured through the use of a selfdeveloped, 4-item questionnaire, initially derived from the Dutch Drinktest (www.drinktest.nl). Answers were recorded on a 5 -point scale.

Organizational Characteristics. Each practice was requested to complete a short questionnaire about practice characteristics. This questionnaire could be filled in by one of the GPs or the practice assistant. We obtained information about practice type (solo practice/group practice) and level of urbanization (large city/city/small urban area).

\section{Sample Size}

The trial was designed to detect a minimal intervention-induced difference of $13 \%$ between the control and intervention groups; it was anticipated that a maximum of $5 \%$ of the patients in the usual care/control group and at least $18 \%$ of the patients in the intervention group would reduce their alcohol consumption from hazardous and harmful to low-risk levels. ${ }^{24} \mathrm{~A}$ total of 64 practices each with at least 10 patients with hazardous and harmful alcohol consumption per practice were needed $(\alpha=$ $0.05 ; \beta=0.80)$. Anticipating a drop-out rate of $25 \%$ of the patients, the aim was to recruit a total of 853 patients with hazardous or harmful alcohol consumption. 


\section{Statistical Analyses}

First, the prevalence of hazardous and harmful alcohol consumption was determined by calculating the number of patients with an AUDIT score $>7$ and $<20 .{ }^{23}$ Descriptive statistics were used to outline the characteristics of this study population using SPSS version 16.0 (IBM/SPSS, Inc, Chicago, IL).

Subsequently, we investigated the effect of the intervention on the dichotomous outcome variable (reduction of alcohol consumption to a low-risk level versus maintenance of hazardous or harmful level of alcohol consumption) by performing a logistic multilevel analysis. Using this technique, regression coefficients can be adjusted for the clustering of observations within one practice that leads to dependency of observations of patients within one practice. In the multilevel analysis 2 levelspatient and practice-were defined. We tested for interaction to see whether the association between condition (intervention vs control) and reduction of alcohol consumption to a low-risk level differed between smokers and nonsmokers. A $P$ value $<.05$ was considered to be statistically significant, except for the interaction analysis, where we used $P<.10$.

Finally, we investigated which patient and practice characteristics were related to the reduction of alcohol consumption to a low-risk level. Therefore, univariate associations between our dependent variable and all the distinguished independent variables were computed. To examine the influence of the most important patient and practice characteristics, a prediction model was built using a backward stepwise regression procedure, starting with all patient and practice characteristics and then eliminating all variables that did not contribute $(P>.10)$ to the multiple multilevel regression model. The intraclass correlation coefficient (ICC), defined as the higher level variance as a percentage of the total variance, was calculated to examine which part of the total variance in the dataset belongs at the practice level (ie, the percentage of the variance that is not different across individual patients but across the practices). Based on the multiple logistic regression model, the ICC was .0001, indicating that the effect of practice was very small. This low ICC suggested that a multilevel model was suitable but not required, enabling us to evaluate the goodness of fit of the model using the Hosmer-Lemeshow statistic. The multilevel analyses were conducted with the statistical program MLwiN version 2.21 (Centre for Multilevel Mod- eling, Institute of Education, London, UK). The estimation method was second-order penalized quasi-likelihood. A $P$ value of $<.05$ was considered significant. Regarding the Hosmer-Lemeshow statistic, a $P$ value of $<.05$ was interpreted as indicating that the model did not fit the data. All outcomes were analyzed by the intention to treat principle, meaning that patients were analyzed in the group to which their practice was originally randomly assigned (regardless of whether the practice actually participated in the intervention).

\section{Results \\ Study Population \\ Practices}

Overall, 2758 general practices were invited to participate; 2676 practices declined. The participating 82 practices $(3.0 \%)$, with 124 active GPs, were randomized. Five practices (one in the intervention group and 4 in the control group) withdrew after randomization but before measurement. This resulted in 37 practices (56 GPs) acting as control group and 40 practices (63 GPs) receiving the intervention. The activities of the program were tailored to the needs and attitudes of the GPs and practices, which resulted in a difference in attendance per GP. Despite several reminders and invitations, 13 GPs did not attend even one educational training or visit by the facilitator. Only half of the intervention practices met the minimal demands made on enrolment (every GP in the practice attend a minimum of one training session and one visit).

\section{Patients}

The GPs from the 77 practices distributed on average 137 patient AUDIT questionnaires per practice; 6775 questionnaires were returned (64\%) and 457 questionnaires were excluded from analyses because of missing data. The control and intervention groups did not significantly differ in the percentages of patients with a hazardous or harmful level of alcohol consumption $(11.6 \%$ and $12.7 \%$, respectively; $P=.17)$. Of the 6318 patients whose AUDIT scores were determined, 712 patients scored above 7 and below 20. Our final sample thus included 366 patients from 34 practices in the control group and 346 patients from 36 practices in the intervention group. Patient characteristics are listed in Table 1. A remarkable difference between 
Table 2. Baseline Characteristics of Practices Providing Care to Patients with Hazardous and Harmful Alcohol Consumption

\begin{tabular}{|c|c|c|c|}
\hline Characteristic & Control Group $(\mathrm{n}=34)$ & Intervention Group $(\mathrm{n}=36)$ & $P^{\star}$ \\
\hline \multicolumn{4}{|l|}{ Practice type } \\
\hline Solo practice & 55.1 & 40.5 & \\
\hline Group practice & 44.9 & 59.5 & $<.01$ \\
\hline \multicolumn{4}{|l|}{ Level of urbanization } \\
\hline Large city (>100,000 inhabitants) & 24.0 & 18.8 & \\
\hline City (30,000-100,000 inhabitants) & 55.7 & 24.6 & \\
\hline Small urban (5000-30,000 inhabitants) & 20.2 & 56.6 & $<.01$ \\
\hline
\end{tabular}

Values provided as percentages.

${ }^{*} P<.05$.

patients registered in control practices and those registered in intervention practices concerned their smoking status: The control group contained a larger proportion of nonsmokers. Table 2 outlines some characteristics of practices in which patients were registered. Compared with the control group, the intervention group contained a smaller proportion of solo practices and a larger part of this group seemed to be established in a small urban area.

Table 1. Baseline Characteristics of Patients with Hazardous and Harmful Alcohol Consumption

\begin{tabular}{|c|c|c|c|}
\hline Characteristic & $\begin{array}{l}\text { Control Group } \\
\quad(\mathrm{n}=366)\end{array}$ & $\begin{array}{l}\text { Intervention Group } \\
\quad(\mathrm{n}=346)\end{array}$ & $P$ \\
\hline Age, years $($ mean $\pm \mathrm{SD})$ & $49.0(16.0)$ & $46.0(16.6)$ & $.02 *$ \\
\hline \multicolumn{4}{|l|}{ Sex } \\
\hline Male & 71.3 & 68.1 & \\
\hline Female & 28.7 & 31.9 & .36 \\
\hline \multicolumn{4}{|l|}{ Household composition } \\
\hline Lives alone & 22.2 & 18.4 & \\
\hline Lives with partner, children, or both & 77.8 & 81.6 & .24 \\
\hline \multicolumn{4}{|l|}{ Education } \\
\hline Basic & 21.8 & 22.1 & \\
\hline Intermediate & 45.5 & 45.3 & \\
\hline High & 32.7 & 32.6 & 1.00 \\
\hline \multicolumn{4}{|l|}{ Smoking status } \\
\hline Nonsmoker & 70.5 & 59.1 & \\
\hline Smoker & 29.5 & 40.9 & $<.01^{*}$ \\
\hline \multicolumn{4}{|l|}{ Comorbidities } \\
\hline Presence of chronic somatic comorbidity without psychiatric comorbidity & 70.5 & 65.9 & .28 \\
\hline Presence of psychiatric comorbidity without chronic somatic comorbidity & 16.9 & 19.7 & .49 \\
\hline Presence of both chronic somatic and psychiatric comorbidity & 12.6 & 14.5 & .59 \\
\hline \multicolumn{4}{|l|}{ Attitudes toward alcohol use } \\
\hline Less alcohol use improves health (yes) & 84.8 & 85.1 & .91 \\
\hline Less alcohol use is not enjoyable (yes) & 59.1 & 53.8 & .17 \\
\hline Less alcohol use complicates relaxing (yes) & 28.4 & 33.3 & .17 \\
\hline \multicolumn{4}{|l|}{ Importance of changing alcohol use } \\
\hline Important & 12.7 & 14.9 & \\
\hline Neutral & 49.0 & 42.1 & \\
\hline Unimportant & 38.3 & 43.0 & .18 \\
\hline
\end{tabular}

Values provided as percentages unless otherwise indicated.

${ }^{*} P<.05$. 


\begin{tabular}{|c|c|c|c|c|c|}
\hline \multirow[b]{3}{*}{ AUDIT Category } & \multicolumn{4}{|c|}{ AUDIT Measurement } & \multirow[b]{3}{*}{$P$} \\
\hline & \multicolumn{2}{|c|}{ At Baseline } & \multicolumn{2}{|c|}{ At 2-Year Follow-up } & \\
\hline & $\begin{array}{l}\text { Control Group } \\
\quad(\mathrm{n}=366)\end{array}$ & $\begin{array}{l}\text { Intervention Group } \\
\quad(\mathrm{n}=346)\end{array}$ & $\begin{array}{l}\text { Control Group } \\
\quad(\mathrm{n}=249)\end{array}$ & $\begin{array}{l}\text { Intervention Group } \\
\quad(\mathrm{n}=217)\end{array}$ & \\
\hline Safe to moderate alcohol use & - & - & 47.0 & 35.5 & $.01^{*}$ \\
\hline Hazardous alcohol use & 89.9 & 91.6 & 47.4 & 58.5 & $.02^{*}$ \\
\hline Harmful alcohol use & 10.1 & 8.4 & 4.0 & 4.6 & .31 \\
\hline Possibly dependent alcohol use & - & - & 1.6 & 1.4 & .84 \\
\hline
\end{tabular}

Values provided as percentages.

${ }^{*} P<.05$.

\section{Changes in Level of Alcohol Consumption}

A substantial proportion of hazardous or harmful alcohol users (41.6\%) reduced their alcohol consumption to a low-risk level at T1 compared with T0. Table 3 shows the changes of level of alcohol consumption between the first and second completion of the AUDIT questionnaire. The proportion of patients with hazardous and harmful alcohol consumption that reduced their alcohol consumption to low-risk levels turned out to be $35.5 \%$ in GP practices in the intervention group compared with $47 \%$ of the GP practices in the control group (odds ratio [OR], 0.62; 95\% confidence interval $[\mathrm{CI}], 0.43-0.90)$. There was no interaction between condition (intervention vs control) and smoking status regarding reduction of alcohol consumption to a low-risk level $(P=$ 0.82 for interaction term). Thus, the association between condition and reduction of alcohol consumption to a low-risk level was the same for smokers and nonsmokers.

\section{Factors Associated with Reduction of Alcohol Consumption to a Low-Risk Level}

The data in Table 4 summarize the results of the multilevel logistic regression analyses regarding the relationship between the reduction of alcohol consumption to a low-risk level and the various patient and practice characteristics. In separate univariate regression analyses, reduction of alcohol consumption to a low-risk level showed an association with sex, smoking status, attitudes toward alcohol use, and urbanization. Female hazardous and harmful alcohol users more often reduced their alcohol consumption to a low-risk level compared with their male counterparts (OR, 1.69; 95\% CI, 1.13-2.55). Smokers, however, less often reduced their alcohol use to a low-risk level compared with nonsmokers (OR, 0.64; 95\% CI, 0.42-0.96). In addition, patients who believed that "less alcohol improves health" and "less alcohol complicates relaxing" were less likely to reduce their alcohol use to a low-risk level compared with those who did not agree with these statements (OR, 0.55; 95\% CI, $0.32-0.96$ and OR, 0.53; 95\% CI, 0.35-0.81, respectively). Interestingly, patients answering in the affirmative to the statement that it is "important to reduce alcohol consumption" less often reduced their alcohol use to a low-risk level compared with those who considered it unimportant to change their alcohol use (OR, 0.42; 95\% CI, 0.22-0.80). Finally, patients registered in practices in small urban areas more often changed their alcohol consumption to a low-risk level compared with patients registered in practices established in large cities (OR, 1.74; 95\% CI, 1.04-2.93).

The multiple analysis showed that older age was positively related with reduction of alcohol consumption to a low-risk level (OR, 1.02; 95\% CI, 1.01-1.03). Furthermore, the multiple analysis showed a stronger association between female sex and alcohol consumption to a low-risk level compared with the univariate analysis (OR, 2.00; 95\% CI, 1.26-3.19). Finally, attitudes toward alcohol use was related to the reduction of alcohol use to a low-risk level in the multiple model. Patients agreeing with the statement "less alcohol use complicates relaxing" less often reduced their alcohol use to a low-risk level compared with those who denied this statement (OR, 0.58; 95\% CI, 0.370.90). In addition, patients answering in the affirmative to the statement that it is important for them to reduce their alcohol consumption less often reduced their alcohol use to a low-risk level 
Table 4. Reduction of Alcohol Use to a Safe Level and the Relationship with Multiple Patient and Practice Characteristics

\begin{tabular}{|c|c|c|c|c|}
\hline \multirow[b]{2}{*}{ Characteristic } & \multicolumn{2}{|c|}{ Univariate Associations* } & \multicolumn{2}{|c|}{ Multiple Analysis $^{\dagger}$} \\
\hline & OR $(95 \% \mathrm{CI})$ & $P$ & OR $(95 \% \mathrm{CI})$ & $P$ \\
\hline Age & $1.01(1.00-1.02)$ & .07 & $1.02(1.01-1.03)$ & $<.01$ \\
\hline Sex & $1.69(1.13-2.55)$ & .01 & $2.00(1.26-3.19)$ & $<.01$ \\
\hline Household composition & $1.06(0.64-1.77)$ & .81 & & \\
\hline \multicolumn{5}{|l|}{ Education } \\
\hline Basic & Reference category & & & \\
\hline Intermediate & $0.88(0.54-1.45)$ & .62 & & \\
\hline High & $0.80(0.48-1.36)$ & .41 & & \\
\hline Smoking status & $0.64(0.42-0.96)$ & .03 & & \\
\hline \multicolumn{5}{|l|}{ Comorbidity } \\
\hline $\begin{array}{l}\text { Presence of chronic somatic comorbidity } \\
\text { without psychiatric comorbidity }\end{array}$ & Reference category & & & \\
\hline $\begin{array}{l}\text { Presence of psychiatric co-morbidity without } \\
\text { chronic somatic comorbidity }\end{array}$ & $0.63(0.31-1.30)$ & .21 & & \\
\hline $\begin{array}{l}\text { Presence of both chronic somatic and } \\
\text { psychiatric comorbidity }\end{array}$ & $0.57(0.27-1.21)$ & .14 & & \\
\hline \multicolumn{5}{|l|}{ Attitudes toward alcohol use } \\
\hline Less alcohol improves health & $0.55(0.32-0.96)$ & .03 & & \\
\hline Less alcohol is not enjoyable & $0.71(0.48-1.04)$ & .08 & & \\
\hline Less alcohol use complicates relaxing & $0.53(0.35-0.81)$ & $<.01$ & $0.58(0.37-0.90)$ & .02 \\
\hline \multicolumn{5}{|l|}{ Importance of changing alcohol use } \\
\hline Unimportant & Reference category & & & \\
\hline Neutral & $0.82(0.55-1.22)$ & .34 & $0.67(0.43-1.05)$ & .08 \\
\hline Important & $0.42(0.22-0.80)$ & .01 & $0.39(0.19-0.80)$ & .01 \\
\hline Practice type & $1.06(0.73-1.53)$ & .77 & & \\
\hline \multicolumn{5}{|l|}{ Level of urbanization } \\
\hline Large city & Reference category & & & \\
\hline City & $1.13(0.66-1.92)$ & .65 & & \\
\hline Small urban & $1.74(1.04-2.93)$ & .04 & & \\
\hline
\end{tabular}

*Data were calculated using multilevel logistic regression analysis to calculate the odds ratio (OR) and 95\% confidence interval (CI) for each patient and practice characteristic independently.

${ }^{\dagger}$ A prediction model was calculated using a backward stepwise regression procedure, starting with all patient and practice characteristics and then eliminating all variables that did not contribute $(P>.1)$ to the model.

compared with those who considered it unimportant to change their alcohol use (OR, 0.39; 95\% CI, 0.19-0.80). The Hosmer-Lemeshow test revealed that the model based on the remaining 4 predictors fit well $(P=.54)$.

\section{Discussion}

Our aim was to assess the effect of implementing a tailored, multifaceted intervention on patients' level of alcohol consumption and to investigate which patient and organizational factors determine reduction of alcohol consumption to a low-risk level. Despite the intensity of the intervention program, the proportion of patients reducing their levels of alcohol consumption to low-risk levels was lower in the intervention group compared with the control group, which was in contrast to our hypothesis. Therefore, we concluded that the intervention did, in fact, increase the odds that patients would continue with hazardous or harmful drinking. This finding is contrary to the evidence that SBIs are effective in reducing alcohol-related problems among persons with hazardous and harmful alcohol consumption in primary health care settings. ${ }^{2,6,8}$ These studies, however, did not assess long-term effects. A possible explanation for our findings that the intervention did not produce the desired outcome on GP level, as described in an earlier article, ${ }^{19}$ or on patient level may be the low participation rate of the GPs. Nonetheless, a sub- 
stantial proportion of patients with hazardous and harmful alcohol consumption (ie, $47.0 \%$ in the control group and $35.5 \%$ in the intervention group) reduced their alcohol use to a low-risk level. These proportions were 3 to 4 times higher than expected from a systematic review-based number needed to treat analysis regarding SBIs. ${ }^{25}$

The phenomenon of regression to the mean may have played a role in the substantial decrease of patients with hazardous and harmful alcohol consumption. More specifically, when patients present to health care providers, it could be for routine health maintenance or it could be for reasons in some way related to consequences of alcohol consumption. As such, their recent consumption at the time of the baseline visit might well have been unusually high, and at follow-up it may have decreased. Furthermore, that this proportion was higher than expected in both groups hints that hazardous and harmful alcohol consumption is not a very constant patient characteristic. Another explanation for the unexpectedly high proportions of patients with hazardous and harmful alcohol consumption who reduced their levels of alcohol consumption to low-risk levels is that social desirability (ie, the patients' desire to be seen as "someone following the advice on reducing alcohol consumption as described in the personal feedback letter") played a role here. Our results also may have been biased by repeated assessment (reactivity): The mere fact of filling out the AUDIT and related measures may have had an effect on patients' drinking behavior. Finally, that this proportion was higher than expected in both groups may imply that the reduction of alcohol consumption to a low-risk level is at least partly attributable to the personal feedback letter, the only patient-directed element in the program that was provided to patients in both groups. Because this feedback letter contained information about only the amount of alcohol consumption and corresponding advice, it may be seen as part of a brief intervention. Therefore, this assumption is consistent with the existing evidence for the efficacy of brief interventions for hazardous and harmful alcohol consumption. ${ }^{2} \mathrm{~A}$ substantial number of patients was lost to followup, but those lost to follow-up were not found to differ markedly between hazardous and harmful alcohol users. Therefore, bias due to loss to follow-up seemed not to have played a large role in our study. Our program, primarily targeted at the attitudes and competences of GPs to improve their SBI activities, did not result in improvement in screening and advice-giving rates. ${ }^{19}$ Because our study revealed no difference in SBI rate between intervention practices and control practices, it is realistic to assume that patient-level effects can be attributed to other factors rather than to the intervention alone. In addition to age and sex, patient attitudes toward alcohol use seemed to be an important determinant of the reduction of alcohol consumption to a low-risk level. Interestingly, we found that patients who considered it important to reduce their alcohol consumption less often reduced their alcohol use to a low-risk level compared with those who considered it unimportant to change their alcohol use. We propose the following explanation for this finding: Drinkers who are aware that their alcohol use has gotten out of hand do not perceive enough benefit from SBIs and need to be offered more intensive or specialized treatment options.

\section{Strengths and Limitations}

To our knowledge, this is the first study reporting the long-term, patient-level effects of a program to engage GPs more in the prevention of alcohol problems. Prior studies of primary care interventions to reduce alcohol-related problems among persons with hazardous and harmful alcohol consumption particularly focused on outcomes regarding changes in GPs' behavior and did not take into account changes in patients' behavior.

Some limitations of this study should be acknowledged. The AUDIT's psychometric properties in the original English version have been well validated. These properties have, however, not been assessed in the Dutch version. Besides that, the appropriateness of traditional, gender-independent AUDIT cutoff scores $(7<$ AUDIT score $<$ 20) used to determine hazardous and harmful alcohol consumption may be subject to criticism. A review of studies of the effects of alternative possible cut points for the AUDIT indicated that the standard value of 8 consistently yielded lower sensitivities and higher specificities for women than for men. ${ }^{26}$ This phenomenon led to the thought that the cut point for female samples should be lowered to a score of 5 or 6 . Several recent studies provide further evidence that 5 may be the best cut point for identifying at-risk drinking among women, with sensitivities ranging from 0.73 to $0.82 .{ }^{27,28}$ Because 
we did not have follow-up data of female patients with an initial AUDIT score of 6 or 7, we were not able to make use of this relatively new evidence on optimal, sex-specific AUDIT cutoff scores during the analysis of our data. A second limitation of our study is that we were not able to reveal insight into possible associations between characteristics of the professional and the reduction of alcohol use to a low-risk level because we collected information about patient registration at the practice level and not at the GP level.

\section{Conclusions and Implications}

Our intervention did not produce the desired outcome at the patient level. Nevertheless, a large proportion of patients with hazardous and harmful alcohol consumption reduced their alcohol use to a low-risk level. The attitude of GPs is a well-known determinant of implementation of SBIs in general practice. ${ }^{29}$ Our study demonstrated that patients' attitudes toward alcohol use also are important determinants of the success of the program. For reaching optimal efficacy of SBIs, it therefore is important to gain insight into patients' attitudes before the intervention starts. Our intervention did not attempt to change patients' attitudes toward alcohol consumption. Patients' attitudes can be influenced through patient-directed interventions such as mass media campaigns and anonymous online treatments. ${ }^{30-33}$ E-learning is an upcoming and proven approach to medical education, which has practical advantages. ${ }^{34-36}$ In future research, more attention should be paid to the effectiveness of the above-mentioned methods instead of intensive implementation strategies aimed at the provider. A stepped approach in which different strategies are used consecutively instead of simultaneously also may be appropriate.

\section{References}

1. Chisholm D, Rehm J, Van OM, Monteiro M. Reducing the global burden of hazardous alcohol use: a comparative cost-effectiveness analysis. J Stud Alcohol 2004;65:782-93.

2. Anderson P, Gual A, Colom J. Alcohol and primary health care: clinical guidelines on identification and brief interventions. Barcelona: Department of Health of the Government of Catalonia; 2005.

3. Rehm J, Mathers C, Popova S, Thavorncharoensap M, Teerawattananon Y, Patra J. Global burden of disease and injury and economic cost attributable to alcohol us and alcohol-use disorders. Lancet 2009; 373:2223-33.

4. World Health Organization (WHO). Reducing risks, promoting healthy life. The World Health Report 2002. Geneva: WHO; 2002.

5. World Health Organization (WHO). Global status report on alcohol 2004. Geneva: WHO; 2004.

6. Anderson P. The effectiveness of general practitioners' advice in reducing the risk of alcohol. In: Lawrence $M$, Neil A, Fowler G, Mant D, editors. Prevention of cardiovascular disease: an evidence-based approach. 2nd ed. Oxford: Oxford University Press; 1996.

7. Fleming MF, Mundt MP, French MT, Manwell LB, Stauffacher EA, Barry KL. Benefit-cost analysis of brief physician advice with problem drinkers in primary care settings. Med Care 2000;38:7-18.

8. Fleming MF, Mundt MP, French MT, Manwell LB, Stauffacher EA, Barry KL. Brief physician advice for problem drinkers: long-term efficacy and benefitcost analysis. Alcohol Clin Exp Res 2002;26:36-43.

9. McAvoy BR. Alcohol education for general practitioners in the United Kingdom - a window of opportunity? Alcohol Alcohol 2000;35:225-9.

10. Owens L, Gilmore IT, Pirmohamed M. General practice nurses' knowledge of alcohol use and misuse: a questionnaire survey. Alcohol Alcohol 2000; 35:259-62.

11. Gual A, Anderson P, Segura L, Colom J. Alcohol and primary health care: training programme on identification and brief interventions. Barcelona: Department of Health of the Government of Catalonia; 2005.

12. Babor TF, Higgins-Biddle JC. Alcohol screening and brief intervention: dissemination strategies for medical practice and public health. Addiction 2000; 95:677-86.

13. Kaner EF, Heather N, McAvoy BR, Lock CA, Gilvarry E. Intervention for excessive alcohol consumption in primary health care: attitudes and practices of English general practitioners. Alcohol 1999;34:559-66.

14. Spandorfer JM, Israel Y, Turner BJ. Primary care physicians' views on screening and management of alcohol abuse: inconsistencies with national guidelines. J Fam Pract 1999;48:899-902.

15. Anderson P, Laurant M, Kaner E, Wensing M, Grol R. Engaging general practitioners in the management of hazardous and harmful alcohol consumption: results of a meta-analysis. J Stud Alcohol 2004; 65:191-9.

16. Anderson P, Jane-Llopis E. How can we increase the involvement of primary health care in the treatment of tobacco dependence? A meta-analysis. Addiction 2004;99:299-312.

17. Funk M, Wutzke S, Kaner E, et al. A multicountry controlled trial of strategies to promote dissemination and implementation of brief alcohol intervention in 
primary health care: findings of a WHO Collaborative Study. J Stud Alcohol 2005;66:379-88.

18. Grol R, Grimshaw J. From best evidence to best practice: effective implementation of change in patients' care. Lancet 2003;362:1225-30.

19. Van Beurden I, Anderson P, Akkermans RP, Grol R, Wensing M, Laurant $M$. Involvement of general practitioners in managing alcohol problems: a randomized controlled trial of a tailored improvement programme. Addiction. 2012 Feb 28. [Epub ahead of print]

20. Fleuren M, Wiefferink K, Paulussen T. Determinants of innovation within health care organizations: literature review and Delphi study. Int J Qual Health Care 2004;16:107-23.

21. Aertgeerts B, Buntinx F, Ansoms S, Fevery J. Screening properties of questionnaires and laboratory tests for the detection of alcohol abuse or dependence in a general practice population. Br J Gen Pract 2001; 51:206-17.

22. Meerkerk GJ, Aarns T, Dijkstra RH, Weisscher P, Njoo K, Boomsma LJ. NHG-Standaard problematisch alcoholgebruik. Huisarts Wet 2005;48:284-5.

23. Babor TF, Higgins-Biddle JC, Saunders JB, Monteiro MG. The Alcohol Use Disorders Identification Test guidelines for use in primary care. Geneva: World Health Organization; 2001.

24. Moyer A, Finney JW, Swearingen CE, Vergun P. Brief interventions for alcohol problems: a metaanalytic review of controlled investigations in treatment-seeking and non-treatment-seeking populations. Addiction 2002;97:279-92.

25. Kaner EF, Beyer F, Dickinson HO, et al. Effectiveness of brief alcohol interventions in primary care populations. Cochrane Database Syst Rev 2007;(2): CD004148.

26. Reinert DF, Allen JP. The Alcohol Use Disorders Identification Test (AUDIT): a review of recent research. Alcohol Clin Exp Res 2002;26:272-9.
27. Gache P, Michaud P, Landry U, et al. The Alcohol Use Disorders Identification Test (AUDIT) as a screening tool for excessive drinking in primary care: reliability and validity of a French version. Alcohol Clin Exp Res 2005;29:2001-7.

28. Reinert DF, Allen JP. The Alcohol Use Disorders Identification Test: an update of research findings. Alcohol Clin Exp Res 2007;31:185-99.

29. Nygaard P, Paschall MJ, Aasland OG, Lun KE. Use and barriers to use of screening and brief interventions for alcohol problems among Norwegian general practitioners. Alcohol Alcohol 2010;45:207-12.

30. Apollonio DE, Malone RE. Turning negative into positive: public health mass media campaigns and negative advertising. Health Educ Res 2009;24(3): 483-495.

31. Perkins HW, Craig DW. A successful social norms campaign to reduce alcohol misuse among college student-athletes. J Stud Alcohol 2006;67:880-9.

32. Riper H, Kramer J, Conijn B, Smit F, Schippers G, Cuijpers P. Translating effective web-based self-help for problem drinking into the real world. Alcohol Clin Exp Res 2009;33:1401-8.

33. Stafstrom M, Ostergren PO, Larsson S, Lindgren B, Lundborg P. A community action programme for reducing harmful drinking behavior among adolescents: the Trelleborg Project. Addiction 2006;101: 813-23.

34. Cook DA, Levinson AJ, Garside S, Dupras DM, Erwin PJ, Montori VM. Internet-based learning in the health professions: a meta-analysis. JAMA 2008; 300:1181-96.

35. Kulier R, Coppus SF, Zamora J, et al. The effectiveness of a clinically integrated e-learning course in evidence-based medicine: a cluster randomized controlled trial. BMC Med Educ 2009;9:21.

36. Riper H, van Straten A, Keuken M, Smit F, Schippers $G$, Cuijpers P. Curbing problem drinking with personalized-feedback interventions: a meta-analysis. Am J Prev Med 2009;36:247-55. 


\section{The tailored intervention offered to the general practices comprised 9 activities, which can} be divided into the following 3 levels:

\section{Professional-directed interventions}

- Distribution of the guideline on problematic alcohol consumption issued by the Dutch college of GPs. $^{22}$

- A reminder card to display on the desk of the GP, with relevant signs, symptoms, and patient characteristics associated with excessive alcohol consumption. Furthermore, the Five Shot Test ${ }^{21}$ was listed as practical tool to question alcohol consumption.

- Educational training sessions: The entire general practice team (including practice assistants and nurses) was invited to participate in the small-scale training sessions (maximum of approximately 10 participants). A minimum of one and maximum of 3 sessions could be attended, tailored to the wishes and needs of the teams. The duration of the sessions was between 2 and 3 hours.

\section{Organization-directed interventions}

- Feedback report: Each practice received written feedback about the proportion of patients with excessive drinking habits according to the AUDIT. The patients were divided into 4 categories: (1) safe to moderate drinker; (2) hazardous drinker; (3) harmful drinker; and (4) possibly dependent drinker.

- Facilitation of the cooperation with local addiction services for support and referral: The local addiction services were invited to join in the first educational training session and to discuss with the GPs both possible treatments and when to refer. Agreements were reached about communication, accessibility, and cooperation.

- Outreach visits by a trained facilitator tailored to needs of practice: The entire practice team was invited to a practice visit by an experienced outreach visitor. The content of the visit was tailored to the wishes and needs of the teams. A minimum of one and maximum of 3 visits were offered, depending on the needs of the practice. The visits took place during daytime and lasted approximately 1 hour.

\section{Patient-directed interventions}

- Patient information letters about alcohol issued by the Dutch College of GPs and leaflets and self-help booklets issued by the National Institute for Health Promotion and Disease Prevention (NIGZ) were offered to the general practices, to be distributed to patients by the GPs.

- Poster in the waiting room: The poster drew the attention to alcohol with the advice to contact the GP or look at the websites of the NIGZ (http://www.nigz.nl) or Netherlands Institute of Mental Health and Addiction (http://www.trimbos.nl) for further information.

- Personal feedback based on their alcohol consumption: The patients received a letter that cited the category (see above) to which they belonged and the corresponding advice. The advice was to consult their GP or to look at the websites of the NIGZ or Trimbos Institute. This was not necessary for patients in category I, and for patients in category IV, the advice to inquire at the local addiction service was added. 